\title{
Fatiga clínicamente relevante en las mujeres con cáncer de mama: prevalencia y factores asociados
}

\author{
Daniela de Araujo Lamino² \\ Cibele Andruciolli de Mattos Pimenta ${ }^{3}$ \\ Patrícia Emilia Braga ${ }^{4}$ \\ Dálete Delalibera Corrêa de Faria Mota5
}

doi: 10.11144/Javeriana.ie 17-2.fcrm

Como citar: Lamino DA, Pimenta CAM, Braga PE, Mota DDCF. Fadiga clinicamente relevante em mulheres com câncer de mama: prevalência e fatores associados. Investig Enferm. Imagen Desarr. 2015;17(1): 157-168. http://dx.doi.org/10.11144/Javeriana.ie17-2.fcrm

1. Traducción del artículo original extraído de la disertación: "Prevalência e fatores associados à fadiga em mulheres com câncer de mama”, presentada a la Escola de Enfermagem da Universidade de São Paulo, Brasil, en 2012.

2. Mestre em Ciências, Escola de Enfermagem, Universidad de São Paulo, Brasil. Bolsista CAPES. Enfermera de investigación clínica, Instituto do Câncer do Estado de São Paulo. Correo electrónico: danilamino@yahoo.com.br

3. Profesora titular, Departamento de Enfermería Médico-Quirúrgica, Universidad de São Paulo, Brasil. Correo electrónico: parpca@usp.br

4. Epidemiologista. Escuela de Enfermería, Universidad de São Paulo, Brasil. Doctora en Salud Pública, São Paulo, Brasil.

5. Profesora-doctora, Facultad de Enfermería, Universidad Federal de Goiás, Brasil. Correo electrónico: dalete.mota@globo.com 


\section{Resumen}

Introducción: la fatiga es un sintoma frecuente en pacientes con cáncer. Objetivo: examinar la prevalencia y los predictores independientes de la fatiga en las mujeres con cáncer de mama. Metodología: se trata de un estudio transversal con una muestra no probabilística de 163 pacientes en acompañamiento ambulatorio. La fatiga fue evaluada por la Escala de Fatiga de Piper. Resultados: fatiga clinicamente relevante (puntuación $\geq 4$ ) estuvo presente en el $31,9 \%$ de la muestra y la intensidad media fue de 6,0 $(\mathrm{DP}=1,3)$. El dolor y la depresión fueron factores independientemente asociados con la fatiga. Conclusiones: la asociación de la fatiga, el dolor y la depresión confirmaron la existencia de una serie de sintomas. El control de la fatiga es poco conocido, pero la depresión y el dolor pueden ser tratados y, tal vez, proporcionar alivio a la fatiga.

Palabras clave: fatiga; prevalencia; factores de riesgo; cáncer de mama; cuidados paliativos

\section{Clinically Relevant Fatigue in Women with Breast Cancer: Prevalence and Associated Factors}

\section{Abstract}

Introduction: Fatigue is a prevalent symptom in cancer patients. Objective: To examine the prevalence and independent predictors of fatigue in women with breast cancer. Methodology: This is a cross-sectional study with a non-probability sample of 163 patients attending an outpatient clinic. Fatigue was assessed by the Piper Fatigue Scale. Results: Clinically relevant fatigue (score $\geq 4$ ) was present in $31.9 \%$ of the sample and the average intensity was $6.0(\mathrm{SD}=1.3)$. Pain and depression were factors independently associated with the fatigue. Conclusions: The fatigue, pain and depression association confirmed the existence of the symptom cluster. The control of fatigue little known, but depression and pain can be treated and perhaps provide relief from fatigue.

Keywords: fatigue; prevalence; risk factors; breast cancer; palliative care

\section{Fadiga clinicamente relevante em mulheres com câncer de mama: prevalência e fatores associados}

\section{Resumo}

Introdução: A fadiga é um sintoma prevalente em pacientes com câncer. Objetivo: Analisar a prevalência e os preditores independentes de fadiga em mulheres com câncer de mama. Metodologia: Trata-se de estudo transversal com amostra não probabilística de 163 pacientes em acompanhamento ambulatorial. Fadiga foi avaliada pela Escala de Fadiga de Piper. Resultados: Fadiga clinicamente relevante (escore $\geq$ 4) esteve presente em $31,9 \%$ da amostra e a intensidade média foi 6,0 (DP = 1,3). Dor e depressão foram fatores independentemente associados à fadiga. Conclusões: A associação fadiga, dor e depressão confirmou a existência de cluster de sintomas. O controle da fadiga é pouco conhecido, mas depressão e dor podem ser tratadas e talvez, proporcionar alívio da fadiga.

Palavras-chave: fadiga; prevalência; fator de risco; neoplasia da mama; cuidados paliativos 


\section{Introducción}

En el mundo, el cáncer de mama es la neoplasia maligna más frecuente entre las mujeres y entre los sintomas que lo acompañan se destaca la fatiga, aún poco investigada en la población brasileña. Varios factores contribuyen a la fatiga relacionada con el cáncer, incluidos aquellos asociados con el tumor, los aspectos psicosociales, y otras morbilidades y síntomas $(1,2)$.

La prevalencia de fatiga en enfermos con cáncer de mama puede variar según la etapa de la enfermedad o el tratamiento realizado. Puede variar entre un $30 \%$ y un $70 \%$ (3); incluso, luego de finalizar el tratamiento, puede afectar a un tercio de las pacientes (2), aproximadamente.

En el caso de cáncer de mama se ha investigado la relación de la fatiga con los síntomas físicos, psicológicos, características del ambiente, características sociodemográficas, tratamiento, medicamentos y alteraciones fisiológicas y genéticas (4-6). Sin embargo, los estudios presentan resultados contradictorios.

En la literatura internacional se ha estudiado la prevalencia y los factores vinculados con la fatiga en pacientes con cáncer; pero en la literatura brasileña existen escasas publicaciones y poco se sabe sobre la prevalencia y cuáles son los elementos que contribuyen a la aparición del síntoma en nuestra población, lo que explica la realización de la presente investigación.

\section{Objetivo}

Los objetivos del estudio fueron analizar la prevalencia y estudiar los factores predictivos independientes relacionados a la fatiga, en mujeres con cáncer de mama.

\section{Método}

Se trata de un estudio transversal. Los datos derivan de un banco de datos, recolectados en la ciudad de São Paulo, en el periodo de julio de 2006 a abril de 2008, en tres servicios de oncología (Clínica de Oncología Médica Privada, Ambulatorio de Oncología del Hospital Brigadeiro y Clinica de Oncología Médica del Hospital Santa Helena).

La muestra, no probabilística, se constituyó por 163 mujeres con cáncer de mama, de manera ambulatoria. Los criterios de inclusión fueron: edad superior a 18 años, escolaridad de mínimo cuatro años de estudio, capacidad de comunicación y comprensión conservadas y ausencia de infección aguda en el momento de la recolección de datos.

La fatiga se evaluó a través de la Escala de Fatiga de Piper-Revisada (0-10), validada en 2009 (7), con un alfa de Cronbach de 0,94. Se evaluaron el dolor y el insomnio, a través de la Escala Visual Numérica (0 a 10); la capacidad funcional, por medio de la Escala de Karnofsky (8), que podia variar de 10 a 100, y la depresión, por medio del Inventario de Depresión de Beck (0-63), validado para el idioma portugués por Gorenstein y Andrade (9).

Se clasificó la fatiga en tres niveles (leve, moderada e intensa), de acuerdo con la distribución de los puntajes en cuartos (cuarto $1=3,2$; cuarto $2=$ 4,8 y cuarto $3=6,2$ ) y el consenso sobre fatiga del National Comprehensive Cancer Network (10), que considera el puntaje de fatiga $\geq 4$ como un evento 
clínicamente significativo, cuando es evaluada en la escala numérica de 0 a 10. Los puntajes que oscilaron entre 0,1 y 3,9 se clasificaron como fatiga leve; de 4,0 a 5,9, como moderada, y de 6,0 a 10, como intensa. Luego se calculó la prevalencia de la fatiga y su respectivo intervalo con una fiabilidad del $95 \%$.

En estudios de corte transversal, la relación entre exposición y resultado se calculó mediante la razón de prevalencia (RP). Cuando la prevalencia es elevada, el odds ratio (OR) no significa una buena aproximación de la RP y su uso es inadecuado $(11,12)$. Considerando la elevada prevalencia de fatiga (puntaje $\geq 4$ ), en el presente estudio $(31,9 \%)$ se calculó la RP y sus respectivos intervalos de confiabilidad (IC95\%) para un análisis de variación única.

Con las variables dependientes en la forma cuantitativa se utilizó el test T de Student o el test no paramétrico de Mann-Whitney, luego de la verificación de la no normalidad, a través del test no paramétrico de Kolmogorov-Smirnov.

Para identificar las variables independientes vinculadas a la fatiga se dio continuidad al análisis con la regresión múltiple de Cox, con variación fuerte (13). En estudios de corte transversal con elevada prevalencia, este modelo se mostró como la alternativa más adecuada para la regresión logística (14). En esta etapa se utilizaron todas las variables que presentaron valores de $p$ inferiores a 0,25 para el análisis de variación única junto a las variables en la forma cualitativa. Los análisis, descriptivos y deductivos, se realizaron en el programa estadístico Stata, versión 9.0, y se adoptó el nivel de importancia del $5 \%$.

\section{Aspectos éticos}

El estudio fue presentado y autorizado por el Comité de Ética de la Escuela de Enfermería de la Universidad de São Paulo (Proceso 511/2005/CEP-EEUSP), así como a los comités del Hospital Santa Helena y del Hospital Brigadeiro.

Se invitó a las pacientes para participar de los estudios, y las que aceptaron firmaron las condiciones de consentimiento establecidas en dos vías.

\section{Resultados}

Entre las mujeres con algún grado de fatiga, o sea, con un puntaje entre 0,1 y 10 (49,7\% de la muestra total del estudio), la intensidad de los sintomas fue de moderada a intensa en el 64,2\%. En esta franja, la intensidad media de la fatiga fue de 6,0 (DP = 1,3; mediana $=6,0 ;$ mínima $=4$; máxima $=9$ ). Ninguna de las pacientes presentó el puntaje máximo de fatiga (10). Al considerar el puntaje de fatiga mayor o igual a cuatro $(\geq 4)$ como aparición de dicho evento, se obtuvo una prevalencia del sintoma del 31,9\% (tablas 1 y 2). 
TABla 1. Razón de prevalencia de fatiga, según variables sociodemográficas, São Paulo (2007-2008)

\begin{tabular}{|c|c|c|c|c|}
\hline Variables & Total & $\begin{array}{c}\text { Fatiga no } \\
\text { (prevalencia) }\end{array}$ & RP (IC95\%) & Valor de $p$ \\
\hline Edad (años) & & & & 0,620 \\
\hline$\leq 45$ & 48 & $18(37,5)$ & 1,00 & \\
\hline $46-64$ & 98 & $29(29,6)$ & $0,79(0,49-1,27)$ & \\
\hline$\geq 65$ & 16 & $5(31,3)$ & $0,83(0,37-1,88)$ & \\
\hline \begin{tabular}{|l} 
Escolaridad \\
(años de estudio; $n=161$ )
\end{tabular} & & & & 0,572 \\
\hline $6-8$ & 13 & $4(30,8)$ & 1,00 & \\
\hline $9-11$ & 51 & $19(37,3)$ & $1,21(0,50-2,96)$ & \\
\hline$\geq 12$ & 97 & $28(28,9)$ & $0,94(0,39-2,25)$ & \\
\hline $\begin{array}{l}\text { Renta familiar en SM } \\
(n=147)\end{array}$ & & & & 0,947 \\
\hline $1-5$ & 41 & $14(34,2)$ & 1,00 & \\
\hline $6-10$ & 35 & $12(34,3)$ & $1,00(0,54-1,88)$ & \\
\hline $11-20$ & 39 & $13(33,3)$ & $0,98(0,53-1,81)$ & \\
\hline$>20$ & 32 & $9(28,1)$ & $0,59(0,41-1,66)$ & \\
\hline $\begin{array}{l}\text { Con compañero } \\
(\mathrm{n}=160)\end{array}$ & & & & 0,839 \\
\hline No & 72 & $24(33,3)$ & 1,00 & \\
\hline Sí & 88 & $28(31,8)$ & $0,95(0,61-1,50)$ & \\
\hline $\begin{array}{l}\text { Trabajo remunerado } \\
(\mathrm{n}=162)\end{array}$ & & & & 0,269 \\
\hline No & 102 & $36(35,6)$ & 1,00 & \\
\hline Sí & 60 & $16(26,7)$ & $0,76(0,46-1,24)$ & \\
\hline $\begin{array}{l}\text { Servicio de atención } \\
(\mathrm{n}=163)\end{array}$ & & & & 0,340 \\
\hline Público & 28 & $11(39,3)$ & 1,00 & \\
\hline Privado & 135 & $41(30,4)$ & $0,77(0,46-1,31)$ & \\
\hline
\end{tabular}

RP: razón de prevalencia; $\mathrm{SM}=$ salario mínimo (considerando al salario mínimo a 380 reales, en el 2007).

NoтA: ninguna de las variables sociodemográficas analizadas presentó vinculación con la fatiga. 
Daniela de Araujo Lamino et al.

TABla 2. Razón de prevalencia de fatiga según variables relacionadas con el tumor y la terapia, São Paulo (2007-2008)

\begin{tabular}{|c|c|c|c|c|}
\hline Variables & Total & $\begin{array}{c}\text { Fatiga no } \\
\text { (prevalencia) }\end{array}$ & RP (IC95\%) & Valor de $\mathbf{p}$ \\
\hline $\operatorname{IMC}(\mathrm{n}=158)$ & & & & 0,360 \\
\hline $18,5-24,9$ & 73 & $20(27,4)$ & 1,00 & \\
\hline $25,0-29,9$ & 59 & $23(39,0)$ & $0,70(0,43-1,15)$ & \\
\hline$\geq 30,0$ & 26 & $8(30,8)$ & $0,79(0,41-1,53)$ & \\
\hline $\begin{array}{l}\text { Hemoglobina } \\
\text { (g/dl; n = 145) }\end{array}$ & & & & 0,030 \\
\hline $12-16$ & 109 & $31(28,4)$ & 1,00 & \\
\hline$<12$ & 36 & $17(47,2)$ & $1,66(1,05-2,62)$ & \\
\hline $\begin{array}{l}\text { Etapa clinica } \\
(\mathrm{n}=124)\end{array}$ & & & & 0,082 \\
\hline I & 31 & $5(16,1)$ & 1,00 & \\
\hline II o III o IV & 93 & $32(34,4)$ & $2,13(0,91-5,01)$ & \\
\hline $\begin{array}{l}\text { Tratamiento actual } \\
(\mathrm{n}=161)\end{array}$ & & & & 0,745 \\
\hline No estaba en tratamiento & 56 & $18(32,1)$ & 1,00 & \\
\hline Quimioterapia & 56 & $18(32,1)$ & $1,00(0,58-1,72)$ & \\
\hline Hormonoterapia & 38 & $11(28,9)$ & $0,90(0,48-1,69)$ & \\
\hline Otros tratamientos* & 11 & $5(45,4)$ & $1,41(0,67-3,00)$ & \\
\hline
\end{tabular}

RP: razón de prevalencia

${ }^{*} \mathrm{QT}+\mathrm{RT}(\mathrm{n}=3) ; \mathrm{QT}+\mathrm{HT}(\mathrm{n}=1) ; \mathrm{RT}+\mathrm{HT}(\mathrm{n}=1) ; \mathrm{HT}+\mathrm{IT}(\mathrm{n}=1) ; \mathrm{RT}(\mathrm{n}=4) ; \mathrm{IM}(\mathrm{n}=1)$.

Se observó que para concentraciones de hemoglobina más bajas hubo una mayor prevalencia de fatiga ( $p=0,030$; mientras que apenas el $28,4 \%$ de las mujeres con índices de hemoglobina adecuados estaban fatigadas. Lo mismo ocurrió en el 47,2\% de aquellas con anemia (tabla 3). 
TABla 3. Razón de prevalencia de fatiga según variables clínicas, São Paulo (2007-2008)

\begin{tabular}{|c|c|c|c|c|}
\hline Variables & Total & $\begin{array}{c}\text { Fatiga } \\
\text { no (prevalencia) }\end{array}$ & RP (IC95\%) & Valor de $p$ \\
\hline $\begin{array}{l}\text { Insomnio } \\
(0-10)\end{array}$ & & & & 0,028 \\
\hline 0 & 84 & $18(21,4)$ & 1,00 & \\
\hline $1-3$ & 12 & $4(33,3)$ & $1,56(0,63-3,83)$ & \\
\hline $4-6$ & 38 & $18(47,4)$ & $2,21(1,30-3,76)$ & \\
\hline $7-10$ & 29 & $12(41,4)$ & $1,93(1,06-3,51)$ & \\
\hline $\begin{array}{l}\text { Dolor } \\
(0-10 ; n=162)\end{array}$ & & & & 0,001 \\
\hline 0 & 86 & $14(16,3)$ & 1,00 & \\
\hline $1-3$ & 22 & $11(50,0)$ & $3,07(1,62-5,81)$ & \\
\hline $4-6$ & 40 & $19(47,5)$ & $2,92(1,63-5,22)$ & \\
\hline $7-10$ & 14 & $7(50,0)$ & $3,07(1,51-6,26)$ & \\
\hline $\begin{array}{l}\text { Depresión } \\
(0-63)\end{array}$ & & & & $<0,001$ \\
\hline $\operatorname{Sin}$ & 134 & $30(22,4)$ & 1,00 & \\
\hline Disforia & 13 & $12(92,3)$ & $4,12(2,90-5,87)$ & \\
\hline Depresión & 16 & $10(62,5)$ & $2,79(1,70-4,58)$ & \\
\hline $\begin{array}{l}\text { Capacidad funcional } \\
(10-100)\end{array}$ & & & & $<0,001$ \\
\hline $90-100$ & 120 & $28(23,3)$ & 1,00 & \\
\hline$<90$ & 43 & $24(55,8)$ & $2,39(1,57-3,64)$ & \\
\hline
\end{tabular}

$\mathrm{RP}$ : razón de prevalencia

Todas las variables clínicas analizadas presentaron estadisticamente una asociación importante con la fatiga. Las pacientes con insomnio, dolor, sintomas depresivos y capacidad funcional reducida mostraron mayores episodios de fatiga (tabla 4). 
Tabla 4. Cálculo de la razón de prevalencia de fatiga en mujeres con cáncer de mama, a través del modelo de regresión múltiple de Cox, São Paulo (2007-2008)

\begin{tabular}{|l|c|c|c|}
\hline \multicolumn{1}{|c|}{ Variables } & RPBR & RPaj (IC95\%) & Valor de $\mathbf{p}$ \\
\hline Depresión (IDB) & 1,06 & $1,06(1,03-1,09)$ & $<0,001$ \\
\hline Dolor & 1,18 & $1,12(1,04-1,21)$ & 0,002 \\
\hline
\end{tabular}

RPBR: razón de prevalencia bruta; RPaj: razón de prevalencia ajustada; IDB: Inventario de Depresión de Beck.

Se observó que al incrementarse en una unidad el puntaje de depresión, hubo un aumento del $6 \%$ en la posibilidad de contraer fatiga y, además, al subir en una unidad la escala de intensidad de dolor, hubo un aumento del $12 \%$ en la posibilidad de contraer fatiga. Variables como índice de hemoglobina, etapa clínica, insomnio y pérdida de la capacidad funcional no presentaron una importancia estadística en el modelo final.

\section{Discusión}

Para identificar la prevalencia de fatiga, en este estudio se adoptó el punto de corte $\geq 4(0-10)$, dado que permite un análisis del síntoma clínicamente relevante. Otros estudios sobre el tema han adoptado el mismo punto de corte, lo que facilita las comparaciones (4,15-18).

De las 163 pacientes con cáncer de mama que conformaron la muestra, el 49,7\% presentó algún grado de fatiga ( $\geq 1$ ). Puntajes $\geq 4$ ocurrieron en el $31,9 \%$ de la muestra, lo que refleja la prevalencia de mujeres con fatiga clínicamente relevante en este estudio.

En las pacientes que presentaron algún grado de fatiga, el síntoma tuvo una intensidad media de 6 , lo que indica fatiga moderada. Otros estudios que evaluaron la fatiga en una escala de 0 a 10 encontraron resultados semejantes en la evaluación de mujeres con tratamiento auxiliar (19) y en pacientes con cáncer de colon-recto (20).

Sin embargo, se notó una gran variación de la presencia de fatiga en investigaciones que utilizaron el mismo punto de corte en la evaluación del sintoma ( 24 ). Durante la radioterapia, apenas el $13 \%$ de las pacientes dijo tener fatiga (15) y en pacientes que realizaron terapia hormonal (4) el resultado fue semejante (15,2\%). En pacientes antes del comienzo de la quimioterapia adyuvante, la prevalencia fue del $28 \%$ (16), pero en pacientes libres del cáncer de mama, esta condición estuvo presente en el $41 \%$ (17) y en el $66,1 \%$ de las muestras (18).

Entre las variables relacionadas con el tumor y la terapia, apenas la hemoglobina presentó una asociación estadísticamente significativa con la fatiga ( $\mathrm{p}<0,030)$, o sea, mujeres con baja concentración de esta presentaron una posibilidad superior al $66 \%$ de presentar fatiga, comparadas con aquellas con cantidades adecuadas (tabla 2).

La anemia es frecuente en pacientes con cáncer, pues contribuye a la caída de las concentraciones de hemoglobina, por la invasión de órganos, por los sangrados tumorales, por los trastornos de absorción y por la insuficiencia 
de la médula ósea. Se encontró solo un estudio que evaluó la hemoglobina en mujeres con cáncer de mama durante la quimioterapia auxiliar (21), y el indice observado fue semejante a nuestro estudio, pues variaba de 12,1 (DP $=1,3)$ a $12,6(\mathrm{DP}=1,3)$. La asociación encontrada entre hemoglobina y fatiga es coherente, dado que un menor número de glóbulos rojos representa menos transporte de oxígeno a la célula y mayor fatiga.

Las variables concentración de hemoglobina, capacidad funcional, depresión, dolor e insomnio se identificaron como posibles factores de riesgo para la fatiga en mujeres con cáncer de mama, y para identificar si serían factores independientes, se sometieron al análisis de regresión múltiple. Para este se insertaron las variables, una a una, en el modelo de regresión y se observó la importancia estadística y su RP. La inserción de las variables en el modelo siguió el orden decreciente de los valores de $p$ : se inició con depresión, seguida de dolor, capacidad funcional, insomnio y hemoglobina. Los factores independientes, asociados con la fatiga en mujeres con cáncer de mama, fueron el dolor y la depresión, pues las demás variables perdieron importancia estadística.

Las mujeres con dolor presentaron una posibilidad de 1,12 más veces de tener fatiga (tabla 4), o sea, un 12\% más, en comparación con aquellas pacientes sin dolor. Este último también fue un indicador de fatiga en otros estudios de pacientes con cáncer de mama, que utilizaron análisis de regresión múltiple $(17,18)$.

Las mujeres con depresión presentaron una posibilidad de 1,06 más veces de tener fatiga (tabla 4), o sea, 6\% más en comparación con las pacientes sin esta morbilidad. La depresión también fue un factor indicador de fatiga en otros estudios, en pacientes con cáncer de mama $(5,22)$.

Al analizar en este estudio la fuerza de las variables sobre la fatiga y el dolor es posible identificar que la Escala Numérica de Evaluación de Dolor posee 11 ítems (de 0 a 10), es decir, el cambio de un punto en la escala aumenta un $12 \%$ la posibilidad de padecer de fatiga. En cambio, en la escala de depresión, que posee 64 ítems (de 0 a 63), el cambio de un punto en el puntaje aumenta un $6 \%$ la posibilidad de padecer fatiga. Aún más importante, después la depresión se convierte en la modificación de la fatiga, en comparación con el dolor.

El análisis de regresión múltiple identifica las variables independientes e importantes para el episodio del resultado de la fatiga, excepto aquellas que parecen relevantes para el suceso, pero que de hecho están "superpuestas" a otras.

Tal vez la exclusión de la variable de índice de hemoglobina se explique por el hecho de que apenas un cuarto de la muestra presentó concentraciones de hemoglobina por debajo de los valores normales, aunque entre las pacientes con anemia el 47,2\% mencionó tener fatiga. A pesar de la elevada prevalencia de fatiga en pacientes con insomnio, en este estudio la variable no se mantuvo en el modelo. No obstante, el sueño fue un indicador de fatiga en otras publicaciones que estudiaron a mujeres con cáncer de mama $(18,22)$. 
La capacidad funcional reducida se asoció con fatiga más intensa en una investigación con pacientes con cáncer en diferentes lugares (23). Sin embargo, en este estudio no ocurrió lo mismo, lo cual puede atribuirse al hecho de que apenas la cuarta parte de la muestra presentó una capacidad funcional reducida, pese a que en estas pacientes la fatiga fue frecuente (tabla 3 ).

\section{Conclusión}

La prevalencia de fatiga clínicamente significativa en mujeres con cáncer de mama fue elevada y la mayoría de ellas presentó sintomas de fatiga, moderada e intensa.

Diversos factores clínicos mostraron asociación con la fatiga y ratificaron la complejidad del síntoma y la existencia de una serie de otros síntomas en oncología. Sin embargo, los factores independientes relacionados con la fatiga fueron el dolor y la depresión. La posibilidad de presentar fatiga fue del $6 \%$ más en las pacientes con depresión y del 12\% más en aquellas con dolor.

Este último y la depresión son tratables en la práctica clínica, lo que tal vez podría aliviar la fatiga, dado que existen pocas intervenciones bien establecidas para su control en pacientes con cáncer.

El presente estudio, pionero en nuestro medio, caracterizó la prevalencia e identificó los factores independientes asociados con la fatiga en mujeres con cáncer de mama, y los resultados encontrados pueden contribuir al perfeccionamiento de la asistencia para estas pacientes.

\section{Conflicto de interés}

Este manuscrito representa un trabajo original, cuyo contenido integral o parcial o sustancialmente semejante no se publicó o sometió a publicación en otro diario, sea en el formato impreso o electrónico. No existe ningún conflicto de interés por parte de los autores, en relación con este manuscrito.

\section{Financiación}

El estudio recibió apoyo financiero de la Coordinación de Perfeccionamiento de Nivel Superior (CAPES).

\section{Referencias}

1. Bower JE. Behavioral symptoms in patients with breast cancer and survivors. J Clin Oncol. 2008;26(5):768-77.

2. Lamino DA, Mota DDCF, Pimenta CAM. Prevalence and comorbidity of pain and fatigue in women with breast cancer. Rev Esc Enferm USP. 2011;45(2):508-14.

3. de M Alcântara-Silva TR, Freitas-Junior R, Freitas NMA, Machado GDP. Fatigue related to radiotherapy for breast and/or gynaecological cancer: a systematic review. J Clin Nurs. 2013:8.

4. Huang X, Zhang Q, Kang X, Song Y, Zhao W. Factors associated with cancer-related fatigue in breast cancer patients undergoing endocrine therapy in an urban setting: a cross-sectional study. BMC Cancer. 2010;10:453. 
5. Minton O, Alexander S, Stone PC. Identification of factors associated with cancer related fatigue syndrome in disease-free breast cancer patients after completing primary treatment. Breast Cancer Res Treat. 2012;136(2):513-20.

6. Rotonda C, Guillemin F, Bonnetain F, Velten M, Conroy T. Factors associated with fatigue after surgery in women with early-stage invasive breast cancer. Oncologist. 2013;18(4):467-75.

7. Mota DDCF, Pimenta CAM, Piper, BF. Fatigue in brazilian cancer patients, caregivers, and nursing students: a psychometric validation study of the Piper Fatigue Scale-Revised. Support Care Cancer. 2009;17:645-52.

8. Karnofsky DA, Burchenal JH. The clinical evaluation of chemotherapeutic agents. In: Mac Leod CM, editor. Evaluation of chemotherapeutic agents. New Jersey: Columbia University Press; 1949. p. 196.

9. Gorenstein C, Andrade L. Validation of a portuguese version of the beck depression inventory and the state-trait anxiety inventory in brazilian subjects. Braz J Med Biol Res. 1996;29:453-7.

10. National Comprehensive Cancer Network (NCCN). Cancer-related fatigue: NCCN Clinical Practice Guidelines in Oncology [internet] [Citado 2010 nov 17]. Disponible en: http://www.nccn.org.

11. Davies HT, Crombie IK, Tavakoli M. When can odds ratios mislead? BMJ. 1998;316(7136):989-91.

12. Barros AJ, Hirakata VN. Alternatives for logistic regression in crosssectional studies: in empirical comparison of models that directly estimate the prevalence ratio. BMC Med Res Methodol. 2003;3:21.

13. Lin DY, Wei LJ. The robust inference for the Cox Proportional Hazards Model. J Am Stat Assoc. 1989;84(408):1074-8.

14. Coutinho LMS, Scazufca M, Menezes PR. Métodos para estimar razão de prevalência em estudos de corte transversal. Rev Saúde Pública. 2008;42(6):992-8.

15. Lavdaniti M, Patiraki E, Dafni U, Katapodi M, Papathanasoglou E, Sotiropoulou A. Prospective assessment of fatigue and health status in Greek patients with breast cancer undergoing adjuvant radiotherapy. Oncol Nurs Forum. 2006;33(3):603-10.

16. Berger AM, Farr LA, Kuhn BR, Fischer P, Agrawal S. Values of sleep/wake, activity/rest, circadian rhythms, and fatigue prior to adjuvant breast cancer chemotherapy. J Pain Symptom Manage. 2007;33(4):398-409.

17. Meeske K, Smith AW, Alfano CM, McGregor BA, McTiernan A, Baumgartner KB, Malone KE, et al. Fatigue in breast cancer survivors two to five years post diagnosis: A HEAL study report. Qual Life Res. 2007;16:947-60.

18. Kim SH, Son BH, Hwang SY, Han W, Yang JH, Lee S, Yun YH. Fatigue and depression in disease-free breast cancer survivors: prevalence, correlates and association with quality of life. J Pain Symptom Manage. 2008;35(6):644-55. 
19. Andrykowski MA, Donovan KA, Laronga C, Jacobsen PB. Prevalence, predictors, and characteristics of off-treatment fatigue in breast cancer survivors. Cancer. 2010;116(24):5740-8.

20. Mota DDCF, Pimenta CAM, Caponero R. Fadiga em pacientes com câncer colorretal: prevalência e fatores associados. Rev Lat-Am Enferm. 2012;20(3):[9 telas].

21. Blair S, Bardwell WA, Podbelewicz-Schuller Y, Mortimer JE. Correlation between hemoglobin and fatigue in women undergoing adjuvant chemotherapy without erythropoietin-stimulating-agent support. Clin Breast Cancer. 2008;8(6):522-26.

22. Dhruva A, Dodd M, Paul SM, Cooper BA, Lee K, West C, et al. Trajectories of fatigue in patients with breast cancer before, during, and after radiation therapy. Cancer Nurs. 2010;33(3):201-12.

23. Cheng KK, Lee DT. Effects of pain, fatigue, insomnia, and mood disturbance on functional status and quality of life of elderly patients with cancer. Crit Rev Oncol Hematol. 2011;78(2):127-37. 